\title{
Mandibular Exercises for the Prevention of Temporomandibular Disorder During Mandibular Advancement Device Therapy in Obstructive Sleep Apnea Patients: a Randomized Controlled Trial
}

Thais de Moura Guimarães ${ }^{1}$, Camila Dinis Tavares ${ }^{2}$, Fernanda R Almeida ${ }^{3}$, Paulo Afonso Cunali ${ }^{4}$, Otávio Ferraz ${ }^{2}$, Cibele Dal Fabbro², Cauby Maia Chaves $\mathrm{Jr}^{5}$, Rowdley Rossi ${ }^{6}$, Sergio Tufik ${ }^{7}$, Lia Bittencourt ${ }^{7}$

\section{Abstract}

Objective: This study aimed to evaluate the efficacy of mandibular exercises for the prevention of temporomandibular disorder (TMD) during Obstructive Sleep Apnea (OSA) treatment with mandibular advancement device (MAD) in patients without TMD symptoms.

Material and methods: Nineteen subjects with mild to moderate OSA were included. Patients were randomized into two groups: a control group and a support therapy group. Both groups received the MAD at $75 \%$ of their maximum protrusion. The support therapy group was instructed to perform mandibular exercises two times per day. Pain was assessed by an analogical pain scale during progressive protrusion at the $4^{\text {th }}, 12^{\text {th }}$ and $16^{\text {th }}$ week. The Research Diagnostic Criteria for Temporomandibular disorders (RDC-TMD) were applied at the initial evaluation and at $16^{\text {th }}$ week. Student's t-test, paired t-test, ANOVA, Friedman's ANOVA and chi-square were performed for statistics analyses.

Results: A total of 19 subjects, 10 in the support therapy group and 9 in the control group completed the protocol. According to the analogical pain scale and the RDC-TMD, none of the patients from either group experienced temporomandibular pain.
1 DDS, MSc, PhD student, Department of Psychobiology, *

2 DDS, MSc, Research at the Sleep Medicine and Biology Division, Department of Psychobiology, *

3 DDS, MSc, PhD, Associate Professor, Division of Orthodontics, Department of Oral Health Sciences, Faculty of Dentistry, The University of British Columbia, Vancouver, Canada

4 DDS, MSc, PhD, Research at the Sleep Medicine and Biology Division, Department of Psychobiology, *

5 DDS, MSc, PhD, Associate Professor, Division of Orthodontics and Pediatric Dentistry, Department of Clinical Dentistry, Federal University of Ceará.

6 DDS, MSc, PhD student, Department of Pneumology, *.

$7 \mathrm{MD}, \mathrm{MSc}, \mathrm{PhD}$, Adjunct Professor, Department of Psychobiology, *

*: Federal University of São Paulo, Brazil

\section{Contact information:}

Thais de Moura Guimarães. Departamento de Psicobiologia, Universidade Federal de São Paulo (UNIFESP).

Address: Napoleão de Barros Street, 925, Vila Clementino, CEP 04021-002, São Paulo-Brasil.

Tel: +55 11-55725092

झ thaisdemouraguimaraes@gmail.com 
Conclusion: Both groups did not develop TMD symptoms, mandible exercise as support therapy did not influence the prevention of TMD.

\section{Keywords}

Oral appliance; Sleep Apnea, Obstructive; Temporomandibular Disorder; Mandibular Exercises; Prevention.

\section{Introduction}

Obstructive sleep apnea (OSA) is characterized by recurrent, partial or complete obstruction of the upper airway during sleep, it causes sleep fragmentation and hypoxemia [1]. In order to normalize the apnea and hypopnea index (AHI), a treatment with MAD (mandibular advancement devices) can be required. MADs are indicated for patients who have primary snoring, mild to moderate OSA or for those patients who do not have a good result with Continuous Positive Airway Pressure (CPAP) or who are poor candidates for CPAP use [2]. In milder cases it can lessen daytime symptoms and improve parameters in quality of life, as well as cardiovascular and neurocognitive function [3-5]. Nonetheless, it has been reported that MAD therapy can provide undesirables effects [6-8].

A very common adverse effect of treatment with $M A D$ is pain and discomfort in the masticatory muscles and/or temporomandibular joint over the short [6-8] and medium term [6]. The pain has been described as mild, transient and tends to disappear over time in patients who use a MAD for long duration [6-9]. This discomfort has been described as a factor related to non-adherence and interruption of MAD treatment [6]. Furthermore, a randomized parallel controlled trial of 2 years MAD and CPAP treatment showed a higher incidence of temporomandibular disorder (TMD) pain at the second month of MAD use [10].

Temporomandibular disorder (TMD) refers to a group of functional and/or structural problems in the masticatory muscles, temporomandibular joints and other associated structures [11]. The most common symptoms of TMD is muscle pain [11]. As a TMD treatment, mandibular exercises are effective to reduce temporomandibular pain and improve coordination, strength and mobility [11, 12]. Therefore, the aim of this study was to investigate the efficacy of mandibular exercises in preventing TMD pain over 4 months of MAD in patients without TMD symptoms in the previous 1 month.

\section{Materials and Methods}

\section{Ethical approval}

All procedures performed in studies involving human participants were in accordance with the ethical standards of the institutional and/or national research committee and with the 1964 Helsinki declaration and its later amendments or comparable ethical standards. Informed consent: Informed consent was obtained from all individual participants included in the study.

\section{Funding}

Fundação de Amparo a Pesquisa do Estado de São Paulo (FAPESP) and Associação Fundo de Apoio a Pesquisa (AFIP) provided financial support in the form of student grant, polissonography exams and oral aplliances. The sponsor had no role in the design or conduct of this research. 


\section{Competing and Conflicting Interests}

All authors certify that they have no affiliations with or involvement in any organization or entity with any financial interest (such as honoraria; educational grants; participation in speakers' bureaus; membership, employment, consultancies, stock ownership, or other equity interest; and expert testimony or patent-licensing arrangements), or non-financial interest (such as personal or professional relationships, affiliations, knowledge or beliefs) in the subject matter or materials discussed in this manuscript.

\section{Patient Selection}

OSA subjects were selected from the center for sleep breathing-related disorders of Association Fund to Encourage Research in São Paulo (SP), Brazil. The current study was in accordance with the Helsinki declaration and was approved by the Ethics Committee of UNIFESP (0162/06). All subjects signed a written informed consent form to participate in the experiment.

The inclusion criteria were: both genders, age between 18 and 65 years old, non obese (body mass index $(\mathrm{BMI}) \leq 30 \mathrm{~kg} / \mathrm{m}^{2}$ ), apnea and hypopnea index (AHI) between 5 and 30 diagnosed by inlaboratory polissomnography and absence of signs or symptoms of TMD based on the Research Diagnostic Criteria for TMD (RDC-TMD) [13], with pain during palpation in less than 3 muscular/joint sites and a negative answer to question "Have you had pain in the face, jaw, temple, in front of the ear or in the ear in the past month?"
The exclusion criteria were: alcoholism, use of psychoactive drugs, habits or professions that lead to sleep deprivation or alteration of the sleep/ wake cycle, other sleep disturbances, previous OSA treatment (MAD, surgery or CPAP), dental status that would compromise the MAD's retention, active periodontal disease, primary dental care needs, protrusive displacement $<5 \mathrm{~mm}$ and limited opening of the mouth.

\section{Protocol}

After selection, 19 subjects were randomized into Support Therapy group (STG) $(n=10)$ or Control group (CG) $(n=9)$. All subjects were assessed by Epworth Sleepiness Scale (ESS) [14], quality of life inventory (Short Form Health Survey/SF-36) (SF-36) [15]; RDC-TMD [16, 17]; panoramic and cephalometric radiographs, dental cast models and photos.

All patients received the same custom-made titrable MAD, the Brazilian Dental Appliance (BRD) [18]. All MADs were set at $75 \%$ of patient's maximum protrusion (max. PT) and subsequently they advanced progressively the mandible $1 \mathrm{~mm}$ per week until the achievement of the maximum comfortable protrusion (MCP). After maximum comfortable protrusion, the subjects returned every 15 days until 16 weeks of the MAD usage.

The Support Therapy (ST) consisted of mandibular exercises twice per day (morning and night), consisting of 3 series of five repetitions for each movement. The figure 1 show the exercises protocol.

Figure 1: Exercises used for coordination and stretching. Movements of opening and closing the mouth with tongue limitation (a), lateral movements against mild hand resistance (b and c), opening the mouth against hand resistance (d), and opening forced assisted by fingers (e).

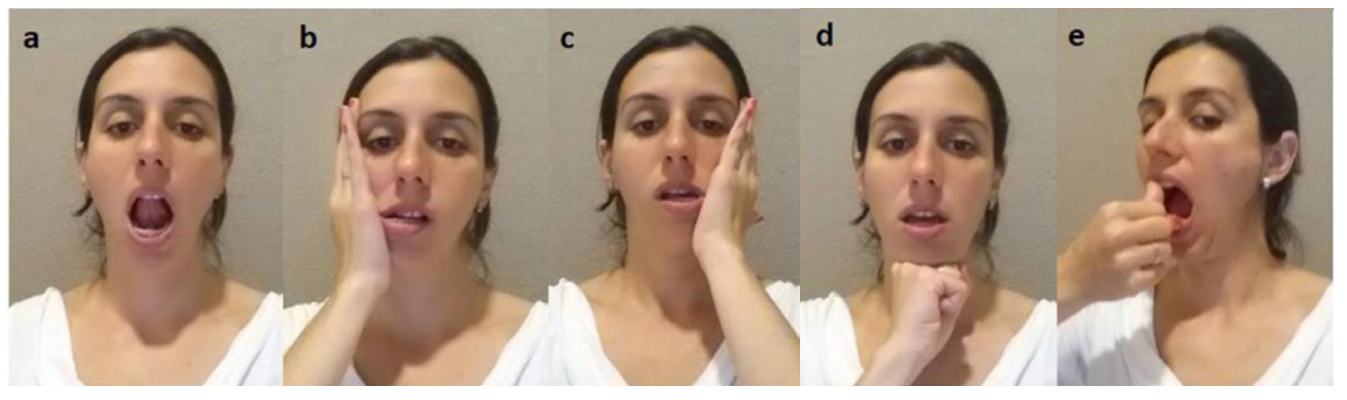


Both groups were instructed to filled out a MAD compliance diary every morning for all 120 days of treatment. The Compliance was determined based on the percentage of nights that the MAD was used.

All patients completed a sleep diary, as well as a MAD compliance diary. At the $16^{\text {th }}$ week of treatment, the subjects filled out the Epworth Sleepiness Scale (ESS) [14], the quality of life inventory (Short Form Health Survey/SF-36) (SF-36) [15], the RDC-TMD [16, 17] and also made a polysomnography. Evaluation of the diaries and the pain scale were undertaken at the weeks 1, 4, 12 and 16 .

Clinical assessment for TMD was made using the Research Diagnostic Criteria for Temporomandibular Disorders (RDC-TMD) [16, 17] was made by one dentist who was blinded to the patient allocation group.

\section{Polysomnography}

A computerized polysomnographic apparatus (EmblaÒ) was used to record the following sleep parameters: electroencephalography (EEG - C3-A2, C4-A1, O2-A1, O1-A2); sub-mentonian and tibial electromyography (EMG); bilateral electrooculography (EOG); and electrocardiography (ECG modified $\mathrm{V} 1$ derivation). Breathing was monitored by a nasal cannula that gauged airflow by pressure transduction. Oral flow was measured by a thermal sensor, and chest and abdominal movements were recorded by a non-calibrated breathing plethysmography. For oxygen hemoglobin saturation, an infrared pulse oximetry reader was placed on the one of the subject's tip fingers. Body position was recorded by a sensor placed over the region of the sternum, and lastly, snoring was measured by a tracheal microphone. According to the previous criteria, the following parameters were evaluated: sleep staging [19], respiratory events, arousals [20] and leg movements [21].

\section{Statistical analysis}

The Statistica computer program (version 6.1; Statsoft, Inc., Tulsa, OK, USA) was used for the statistical analysis. For normally distributed data, we adopted Student's t-test for independent samples, the paired t-test for comparisons within the same group, or ANOVA for repeated measures to compare between evaluation times. When data did not present a normal distribution, non-parametric test was adopted: Friedman's ANOVA for evaluation of visual analog scale pain. The chi-square test was used to compare qualitative variables. $P$ values $<0.05$ were considered significant.

\section{Results}

A total of 87 consecutive patients with OSA (41 M/46 F) referred for treatment with MADs were evaluated, and 45 of them were excluded because of TMD pain. Of the 42 remaining subjects, 20 were excluded due to periodontal disease or insufficient teeth. Three subjects dropped out, one male in the support therapy group because of personal problems; and one male and one female in the control group, who did not give reasons for dropping out. The final sample was composed of 19 subjects, being 5 women (26.3\%) and 14 men (73.7\%), with a mean age of $44 \pm 9$ years old and a mean BMI of $25 \pm 3 \mathrm{~kg} / \mathrm{m}^{2}$.

After randomization, the Control Group (CG) consisted of 9 subjects, and the Support Therapy Group (STG) consisted of 10 subjects. The anthropomorphic characteristics of baseline are described in Table 1; the only differences between groups at

Table 1. General characteristics of the control and support therapy groups at baseline.

\begin{tabular}{|l|c|c|}
\hline & $\begin{array}{c}\text { Control } \\
\text { Group }\end{array}$ & $\begin{array}{c}\text { Support } \\
\text { Therapy } \\
\text { Group }\end{array}$ \\
\cline { 2 - 3 } & $\mathrm{n}=9$ & $\mathrm{n}=10$ \\
\hline Gender (M/F) & $7 / 2$ & $7 / 3$ \\
\hline Age (years) & $42.3 \pm 3.2$ & $45.6 \pm 2.7$ \\
\hline BMI (kg/m $\left.{ }^{2}\right)$ & $26.1 \pm 0.7$ & $24.9 \pm 1.0$ \\
\hline Epworth scale & $9.6 \pm 2.2$ & $11.4 \pm 1.7$ \\
\hline AHI & $13.5 \pm 0.8$ & $17.3 \pm 2.3$ \\
\hline
\end{tabular}




\begin{tabular}{|c|c|c|}
\hline & $\begin{array}{l}\text { Control } \\
\text { Group }\end{array}$ & $\begin{array}{c}\text { Support } \\
\text { Therapy } \\
\text { Group }\end{array}$ \\
\hline & $n=9$ & $n=10$ \\
\hline \multicolumn{3}{|l|}{ SF-36 } \\
\hline Physical function & $61.0 \pm 13.2^{*}$ & $88.6 \pm 2.9 *$ \\
\hline $\begin{array}{l}\text { Role limitation due to physical } \\
\text { problems }\end{array}$ & $50.1 \pm 13.4$ & $77.7 \pm 10.4$ \\
\hline Pain & $78.1 \pm 7.7$ & $74.8 \pm 7.9$ \\
\hline General health perception & $54.5 \pm 12.2$ & $72.5 \pm 5.9$ \\
\hline Vitality & $61.9 \pm 9.8$ & $63.6 \pm 7.6$ \\
\hline Social function & $73.8 \pm 8.8$ & $77.8 \pm 9$ \\
\hline $\begin{array}{l}\text { Role limitation due to } \\
\text { emotional problems }\end{array}$ & $70.8 \pm 16.0$ & $78.6 \pm 12.1$ \\
\hline Mental health & $78.9 \pm 5.9$ & $68.7 \pm 4.5$ \\
\hline \multicolumn{3}{|c|}{$\begin{array}{r}\text { Data described in mean and standard deviation. } \\
\text { BMI = Body mass index, } \mathrm{AHI}=\text { apnea/hypopnea index, SF- } \\
36=\text { Short Form Health Survey; (Student's t-test): } \\
{ }^{*}=\mathrm{p}<0.05 \text { between the groups. (chi-square test): } \\
\quad \#=\mathrm{p}<0.05 \text { between the groups. }\end{array}$} \\
\hline
\end{tabular}

baseline was Physical functioning domain of SF36 and the lower score of CG compared to STG (Table 1).

At the baseline comparison, the polysomnography variables from both groups did not differ. The AHI was significantly reduced between baseline and the final follow-up in both groups (Table 2).

There was not any significant difference between groups or between assessments regarding to MAD compliance in the 4 appointments (weeks 1, 4, 12 and 16) (table 3). No significant difference was found in exercise compliance over time.

Table 4 describes the results of the Epworth Sleepiness Scale and SF-36 between the 2 groups, comparing the baseline and final follow-up. The STG presented a statistically significant reduction in Epworth score after treatment. The SF-36 changes with treatment were observed in Physical functio-

Table 2. Comparison of baseline and final polysomnographic variables between the control group and support therapy group.

\begin{tabular}{|l|c|c|c|c|}
\hline & $\begin{array}{c}\text { Control group } \\
\text { (Baseline) }\end{array}$ & $\begin{array}{c}\text { Control group } \\
\text { (Final) }\end{array}$ & $\begin{array}{c}\text { Support therapy group } \\
\text { (Baseline) }\end{array}$ & $\begin{array}{c}\text { Support therapy group } \\
\text { (Final) }\end{array}$ \\
\hline $\mathrm{AHI}$ & $13.5 \pm 0.8^{* *}$ & $6.7 \pm 1.8^{* *}$ & $17.3 \pm 2.3^{*}$ & $7.5 \pm 1.0^{*}$ \\
\hline Mean $\mathrm{SpO}_{2}$ & $95.5 \pm 1.3$ & $96 \pm 1,1$ & $94.5 \pm 1.1$ & $96 \pm 0.8$ \\
\hline Lowest $\mathrm{SpO}_{2}$ & $88.1 \pm 4.4$ & $90.4 \pm 1,7$ & $87 \pm 4.5$ & $89.4 \pm 4.2$ \\
\hline $\mathrm{SE}(\%)$ & $81.0 \pm 3.8$ & $86.9 \pm 2.4$ & $84.0 \pm 2.7$ & $87.8 \pm 2.7$ \\
\hline E1 (\%) & $4.6 \pm 0.9$ & $5.1 \pm 1.3$ & $4.8 \pm 1.5$ & $3.2 \pm 0.6$ \\
\hline E2 (\%) & $56.1 \pm 3.9$ & $55.4 \pm 2.3$ & $55.4 \pm 2.8$ & $58.5 \pm 2.2$ \\
\hline E3 (\%) & $21.8 \pm 4.3$ & $21.3 \pm 1.7$ & $17.3 \pm 2.5$ & $17.4 \pm 2.0$ \\
\hline REM (\%) & $17.6 \pm 2.2$ & $18.2 \pm 2.5$ & $19.3 \pm 1.8$ & $22.1 \pm 1.5$ \\
\hline Arousal index & $12.6 \pm 2.4$ & $13.2 \pm 2.2$ & $14.6 \pm 2.9$ & $11.9 \pm 1.7$ \\
\hline
\end{tabular}

Data described in mean SD .AHI = apnea/hypopnea index, SE= sleep efficiency.CG=Control group; STG=Support therapy group.(Paired t-test): ${ }^{*}=\mathrm{p}<0.05$ between baseline and after treatment in support therapy group. (Paired t-test): $* *=p<0.05$ between baseline and after treatment in control group.

Table 3. MAD compliance and exercise compliance between control and support therapy group. Data expressed in percentage of the nights.

\begin{tabular}{|c|c|c|c|c|c|}
\hline & Groups & $\begin{array}{c}1^{\text {st }} \text { week MAD } \\
\text { of } 75 \%\end{array}$ & $4^{\text {th }}$ week & $12^{\text {th }}$ week & $\begin{array}{l}16^{\text {th }} \text { week } \\
\text { maximum } \\
\text { protrusion }\end{array}$ \\
\hline Control group & MAD compliance & $87.0 \pm 0.8$ & $88.5 \pm 0.8$ & $96.3 \pm 0.8$ & $92.0 \pm 0.8$ \\
\hline \multirow{2}{*}{ Support therapy group } & MAD compliance & $87.5 \pm 0.7$ & $84.6 \pm 0.7$ & $81.0 \pm 0.7$ & $80.9 \pm 0.7$ \\
\hline & Exercise compliance & $80.1 \pm 0.7$ & $75.5 \pm 0.7$ & $60.2 \pm 0.7$ & $64.8 \pm 0.7$ \\
\hline
\end{tabular}


Table 4. Questionnaires at baseline and after intervention for both groups.

\begin{tabular}{|c|c|c|c|c|}
\hline & $\begin{array}{l}\text { Control group } \\
\text { (Baseline) }\end{array}$ & $\begin{array}{l}\text { Control group } \\
\text { (Final) }\end{array}$ & $\begin{array}{l}\text { Support therapy group } \\
\text { (Baseline) }\end{array}$ & $\begin{array}{l}\text { Support therapy group } \\
\text { (Final) }\end{array}$ \\
\hline Epworth scale & $9.6 \pm 2.2$ & $8.0 \pm 1.8$ & $11.4 \pm 1.7^{*}$ & $8.4 \pm 1.1^{*}$ \\
\hline \multicolumn{5}{|l|}{ SF-36 } \\
\hline Physical functioning & $61.0 \pm 13.2^{* *}$ & $72.3 \pm 11.7 * *$ & $88.6 \pm 2.9$ & $85.7 \pm 6.3$ \\
\hline Role limitation due to physical problems & $50.1 \pm 13.4$ & $78.1 \pm 14.5$ & $77.7 \pm 10.4$ & $79.5 \pm 10.0$ \\
\hline Pain & $78.1 \pm 7.7$ & $75.0 \pm 6.6$ & $74.8 \pm 7.9$ & $76.9 \pm 5.1$ \\
\hline General health perception & $54.5 \pm 12.2$ & $72.6 \pm 6.5$ & $72.5 \pm 5.9 *$ & $81.1 \pm 4.0 *$ \\
\hline Vitality & $61.9 \pm 9.8$ & $66.9 \pm 9.1$ & $63.6 \pm 7.6$ & $71.4 \pm 8.0$ \\
\hline Social functioning & $73.8 \pm 8.8$ & $75.0 \pm 12.3$ & $77.8 \pm 9$ & $89.0 \pm 6.0$ \\
\hline Role limitation due to emotional problems & $70.8 \pm 16.0$ & $70.8 \pm 13.3$ & $78.6 \pm 12.1$ & $78.8 \pm 1.3$ \\
\hline Mental health & $78.9 \pm 5.9$ & $67.5 \pm 9.7$ & $68.7 \pm 4.5$ & $75.3 \pm 4.3$ \\
\hline
\end{tabular}

Data described in mean SD.(Paired t-test): * $=p<0.05$ between baseline and after treatment in Support therapy group. (Paired t-test): ${ }^{* *}=p<0.05$ between baseline and after treatment in Control group.

Table 5. Comparison of visual analog scale pain values between control and support therapy group.

\begin{tabular}{|l|c|c|c|c|}
\hline & $1^{\text {st }}$ week MAD of $75 \%$ & $4^{\text {th }}$ week & $1^{\text {th }}$ week & $1^{\text {th }}$ week maximum protrusion \\
\hline Control group & $0.0 \pm 0.0$ & $0.3 \pm 0.3$ & $0.0 \pm 0.0$ & $0.0 \pm 0.3$ \\
\hline Support therapy group & $0.2 \pm 0.2$ & $0.1 \pm 0.1$ & $0.1 \pm 0.1$ & $0.0 \pm 0.0$ \\
\hline \multicolumn{2}{|r}{ (Friedman's ANOVA): ${ }^{*}=p<0.05$ between times in each group. (Friedman's ANOVA): ** $=$ p<0.05 between groups at } \\
each time
\end{tabular}

ning for the CG group and in general health perception for the STG.

The visual analog scale of pain at the first week (at $75 \%$ of maximum mandible advancement) in either group and when compared with the values at the $4^{\text {th }}, 12^{\text {th }}$, and $16^{\text {th }}$ weeks showed no significant differences, the groups did not present any differences between each other at any time (Table 5). Regarding the evaluation of RDC-TMD, we observed that none of the patients developed TMD pain.

\section{Discussion}

This is the first randomized controlled clinical trial which proposed to use jaw exercises to prevent temporomandibular pain in individuals who use MAD and had no previous TMD. This study is important because temporomandibular pain is a very common side effect related to $\operatorname{MAD}[8,22,23]$ and it is a factor of non-adherence and interruption of
MAD treatment7. While there is a tendency of prescribing exercises to patients, we understand with our current study that, for patients without previous $T M D$, there is no need for exercises and we can decrease the burden on patients undergoing MAD treatment.

In our study, both groups did not show any development of TMD pain. Many studies which evaluated side effects did not assess previous TMD pain or did not exclude this condition of the sample based on a strict evaluation criteria, the RDC-TMD. The previous assessment of temporomandibular joint is important since the etiology of TMD is multifactorial [24] and the frequency of the presence of TMD has been described to be as high as $52 \%$ of untreated sleep apnea patients [25].

Few studies assessed systematically TMD by RDCTMD in a short MAD follow up $[6,8,10]$. One study showed $24 \%$ of subjects developed TMJ pain when using MAD for 2 months compared with $6 \%$ of 
those using CPAP [10]. Another study did not show increase of TMD pain at an average of 118 days of treatment with MAD, but showed increase of TMD pain in analyzed dropout at 208 days [6] and one research realized in 2010 did not find increase in TMD pain at week 6 and month 6 after titration of MAD [8].

In our study, no dropouts occurred because of pain, and none of the subjects felt temporomandibular pain. We assessed TMD by RDC-TMD at 4 months of treatment, which is similar to 118 days follow up of a study realized in 2013 [6]. On the other hand, our follow up is in a different time comparing to 2 months of follow up of another study [10]. However, we used a visual analog scale at the 2 months interval, at the time of 118 days follow up [6], and we did not show increase of TMD pain in any of the groups. One hypothesis of this difference in the findings is that our sample consisted of patients who did not have TMD for 30 days before treatment with MAD.

Some studies have been published about exercises to aider MAD treatment $[26,27]$. It has been showed that the positive effects of two kinds of mandibular exercises on occlusal contacts and bite force in patients who used MAD [26]. The effectiveness of mandibular exercises to control pain in patients who were submitted to treatment with MADs and already had diagnosis of TMD has also been demonstrated [27]. In our study, mandibular exercises were used to prevent temporomandibular pain. However, we did not find any pain related to MAD short-term, this findings need to be confirmed in a larger sample size with similar baseline characteristics.

According to the study's results, the MAD population did not show TMD pain and the exercises were not required to prevent TMD. However, mandibular exercises therapy improved general health perception and sleepiness and control group improved physical functioning. Studies showed mandibular exercises and speech therapy improved quality of life in CPAP and MAD therapy $[27,28]$. There is an improvement on CPAP compliance and functional capacity domain after speech therapy associated with CPAP compared to patients who use only CPAP [28]. In pain patients, mandibular exercises are effective to improve complain to MAD and improve general health perception, role limitation due to emotional problems, mental health compared with placebo therapy [27].

Therefore, patients who did not have previous TMD appear to be good candidates for treatment with MAD, without the need of mandibular exercise as a support therapy to prevent pain or to improve adherence, but others studies are necessary to confirm this statement. However, the results allow us to suggest that OSA patients undergoing MAD therapy require specific evaluations for $T M D$, which could influence treatment protocol.

Our study is the first trial to systematically and carefully assess the impact of mandibular exercises on the temporomandibular complex in patients undergoing MAD treatment who had no TMD symptoms in the previous 1 month. Although, it has some limitation, as the sample size was small, the results were highly consistent in our sample. Also the diagnosis of TMD by RDC-TMD was made by only one examiner, still we have assured that the examiner was blinded to the group allocation.

\section{Conclusion}

The control group and the exercise group did not show any development of TMD symptoms over 4 months of MAD use and mandible exercise as support therapy did not influence the prevention of TMD.

\section{Abbreviation \\ AFER: Association Fund to Encourage Research \\ AHI: Apnea and hypopnea index \\ BRD: Brazilian Dental Appliance \\ CG: Control group}


CPAP: Continuous Positive Airway Pressure

ECG: Electrocardiography

EEG: Electroencephalography

EMG: Electromyography

EOG: Electrooculography

ESS: Epworth Sleepiness Scale.

Fig.: Figure

MAD: Mandibular advancement devices

OSA: Obstructive sleep apnea

RDC -TMD: Research Diagnostic Criteria for Temporomandibular disorder

SF- 36: Short Form Health Survey

ST: Support therapy

STG: Support therapy group

TMD: Temporomandibular disorder

\section{Acknowledgments}

The authors thank Fundação de Amparo a Pesquisa do Estado de São Paulo (FAPESP) and Associação Fundo de Apoio a Pesquisa (AFIP).

\section{References}

1. American Academy of Sleep Medicine. The International Classification of sleep disorders: Diagnostic and coding manual 2nd ed. Weztchester, NY: American Academy of Sleep Medicine; 2005

2. Kushida CA, Morgenthaler TI, Littner MR, et al. Practice parameters for the treatment of snoring and Obstructive Sleep Apnea with oral appliances: an update for 2005. Sleep. 2006; 29: $240-3$

3. Gagnadoux F, Fleury B, Vielle B, et al. Titrated mandibular advancement versus positive airway pressure for sleep apnoea. Eur Respir J. 2009; 34: 914-20.

4. Gotsopoulos H, Kelly JJ, Cistulli PA. Oral appliance therapy reduces blood pressure in obstructive sleep apnea: a randomized, controlled trial. Sleep. 2004; 27:934-41.

5. Phillips $\mathrm{CL}$, Grunstein RR, Darendeliler MA, et al. Health outcomes of continuous positive airway pressure versus oral appliance treatment for obstructive sleep apnea: a randomized controlled trial. Am J Respir Crit Care Med 2013;187:879-87.

6. Perez $C V$, de Leeuw $R$, Okeson JP, et al. The incidence and prevalence of temporomandibular disorders and posterior open bite in patients receiving mandibular advancement device therapy for obstructive sleep apnea. Sleep Breath. 2013; 17: 323-32.
7. Fransson AMC, Tegelberg A, Johansson A, Wenneberg B. Influence on the masticatory system in treatment of obstructive sleep apnea and snoring with a mandibular protruding device: A 2-year follow-up. Am J Orthod Dentofacial Orthop. 2004; 126: 687-693

8. Martínez-Gomis J, Willaert E, Nogues L, Pascual M, Somoza $\mathrm{M}$, Monasterio $\mathrm{C}$. Five years of sleep apnea treatment with a mandibular advancement device. Side effects and technical complications. Angle Orthod. 2010; 80:30-6.

9. Giannasi LC, Almeida FR, Magini M, César J, De Oliveira M, Bussadori SK. Systematic assessment of the impact of oral appliance therapy on the temporomandibular joint during treatment of obstructive sleep apnea: long-term evaluation. Sleep Breath. 2009; 13: 375-381.

10. Doff MHJ, Veldhuis SKB, Hoekema $A$, et al. Long-term oral appliance therapy in obstructive sleep apnea syndrome: a controlled study on temporomandibular side effects. Clin Oral Investig. 2012; 16: 689-97.

11. De Leeuw R Orofacial pain: guidelines for assessment, diagnosis and management. 4nd ed. Chicago, IL:Quintessence Publishing; 2008.

12. Mcneely ML, Olivo SA, David J A. Systematic Review of the Effectiveness of Physical Temporomandibular Disorders. Phys Ther. 2006; 86(5):710-25.

13. Dworkin SF, LeResche L. Research diagnostic criteria for temporomandibular disorders: review, criteria, examinations and specifications, critique. J Craniomandib Disord. 1992; 6:30155.

14. Bertolazi AN, Fagondes SC, Hoff LS, Pedro VD, Menna Barreto SS, Johns MW. Portuguese-language version of the Epworth sleepiness scale: validation for use in Brazil. J Bras Pneumol. 2009; 35: 877-83.

15. Severo M, Santos AC, Lopes C, Barros H. [Reliability and validity in measuring physical and mental health construct of the Portuguese version of MOS SF-36]. Acta Med Port. 2006; 19:281-7.

16. Pereira Jr. HKFJ, Favilla EE, Dworkin SF. Research Diagnostic Criteria for Temporomandibular Disorders (RDC/TMD); formal translation to portuguese. J Bras Clin Odontol Integr. 2004; 8:384-95

17. Dworkin JF, Sherman J, Mand L, Ohrbach R, LeResche L, Truelove E. Reliability, validity, and clinical utility of the research diagnostic criteria for Temporomandibular Disorders Axis II Scales: depression, non-specific physical symptoms, and graded chronic pain. J Orofac Pain. 2002; 16:207-20.

18. Dal-fabbro C, Maia C, Bittencourt LR, Tufik S. Clinical and polysomnographic assessment of the BRD Appliance in the treatment of Obstructive Sleep Apnea Syndrome. Dental Press J. Orthod. 2010; 15:107-117. 
19. Rechtschaffen A, Kales A. A manual of standardized terminology, techniques and scoring sytem for sleep stages of human subjects. Los Angeles,CA: Brain Information Service/ Brain Research Institute/UCLA. 1968.

20. Atlas Task Force of the American Sleep Disorders Association. EEG arousals: scoring rules and examples: a preliminary report from the Sleep Disorders. Sleep. 1992; 15:173-84.

21. The Atlas Task Force. Recording and scoring leg movements. Sleep. 1993; 16:748-59.

22. Deane SA, Cistulli PA, Ng AT, Zeng B, Petocz P, Darendeliler MA. Comparison of mandibular advancement splint and tongue stabilizing device in obstructive sleep apnea: a randomized controlled trial. Sleep. 2009; 32:648-53.

23. Hammond RJ, Gotsopoulos H, Shen G, Petocz P, Cistulli PA, Darendeliler MA. A follow-up study of dental and skeletal changes associated with mandibular advancement splint use in obstructive sleep apnea. Am J Orthod Dentofacial Orthop. 2007; 132:806-14.

24. Poveda Roda R, V Bagan J, Díaz Fernández JM, Hernández Bazán S, Jiménez Soriano Y. Review of temporomandibular joint pathology. Part I: classification, epidemiology and risk factors. Med Oral Patol Oral Cir Bucal. 2007; 12:E292-8.

25. Cunali PA, Almeida FR, Santos CD, Valdrighi NY, Nascimento LS, Dal'Fabbro C, Tufik S, Bittencourt LR. Prevalence of temporomandibular disorders in obstructive sleep apnea patients referred for oral appliance therapy. J Orofac Pain. 2009; 23:339-44.

26. Otsuka R, Almeida FR, Lowe AA. The effects of oral appliance therapy on occlusal function in patients with obstructive sleep apnea: a short-term prospective study. Am J Orthod Dentofacial Orthop. 2007;131:176-83.

27. Cunali PA, Almeida FR, Santos CD, et al. Mandibular exercises improve mandibular advancement device therapy for obstructive sleep apnea. Sleep Breath. 2011; 15:717-27.

28. Diaferia G, Badke L, Santos-Silva R, Bommarito S, Tufik S, Bittencourt L. Effect of speech therapy as adjunct treatment to continuous positive airway pressure on the quality of life of patients with obstructive sleep apnea. Sleep Med. 2013; 14:62835.

\section{Publish in International Archives of Medicine}

International Archives of Medicine is an open access journal publishing articles encompassing all aspects of medical science and clinical practice. IAM is considered a megajournal with independent sections on all areas of medicine. IAM is a really international journal with authors and board members from all around the world. The journal is widely indexed and classified Q1 in category Medicine. 
